\title{
Some New Aspects of Metric Fixed Point Theory
}

\author{
Khalil Javed $\mathbb{D}^{1},{ }^{1}$ Fahim Uddin, ${ }^{1,2}$ Hüseyin Ișılk $\mathbb{D},{ }^{3}$ Tareq M. Al-shami $\mathbb{D}^{4}{ }^{4}$ Faizan Adeel, ${ }^{1}$ \\ and Muhammad Arshad ${ }^{1}$ \\ ${ }^{1}$ Department of Mathematics and Statistic, International Islamic University, Islamabad, Pakistan \\ ${ }^{2}$ NUIST Reading Academy 219 Ningliu Road, Nanjing, Jiangsu 210044, China \\ ${ }^{3}$ Department of Engineering Science, Bandırma Onyedi Eylül University, 10200 Bandırma, Balıkesir, Turkey \\ ${ }^{4}$ Department of Mathematics, Sana'a University, Sana'a, Yemen
}

Correspondence should be addressed to Hüseyin Işı; huseyin.isik@tdtu.edu.vn and Tareq M. Al-shami; tareqalshami83@gmail.com

Received 19 May 2021; Accepted 29 June 2021; Published 10 August 2021

Academic Editor: Sergey Shmarev

Copyright (c) 2021 Khalil Javed et al. This is an open access article distributed under the Creative Commons Attribution License, which permits unrestricted use, distribution, and reproduction in any medium, provided the original work is properly cited.

In this manuscript, we prove fixed point results in $R_{m}$-metric spaces endowed with an amorphous binary relation. Moreover, we give an example to highlight the utility of our main results. Finally, we apply our result to examine the existence and uniqueness of the solution for a Fredholm integral equation.

\section{Introduction and Preliminaries}

In metric fixed point theory, Banach contraction mapping principle [1] is one of the most fundamental tools to investigate the existence and uniqueness of solutions for contraction maps in a complete metric space. Since the appearance of this classical result, researchers have taken keen interest in generalizing and extending this result in different ways, either by improving contraction conditions or by relaxing the axioms of metric space. One may recall the existing notions of, namely, partial metric space [2], partial $b$-metric space [3], partially ordered $S$-metric space $[4,5]$, partial symmetric space [6], partial JS-metric space [7], metriclike space [8], $b$-metric space [9], rectangular metric space [10], and several others. In 2014, Asadi et al. [11] extended the concept of partial metric space and introduced the notion of $m$-metric spaces to investigate fixed point. This concept was extended in many different ways, such as $m_{b}$ -metric space [12], $m_{v}$-metric space [13, 14], and rectangular $m$-metric space [15]. Also in this sequel, Patle et al. [16] extended the notion of $m$-metric by proving fixed point results for Nadler and Kannan type set valued mappings in $m$-metric spaces.

Recently, Gordji et al. [17] introduced the notion of orthogonal sets and gave a new extension for the classical Banach contraction principle; more details can be found in [18-20]. Utilizing the structure of orthogonal metric spaces, which appeared in $[18,19]$, and the binary relation used with a metric, in [21], Ali et al. [22] introduced the notion of $R$ -partial $b$-metric space and proved fixed point results in this space. Recently, Javed et al. [23] studied fixed point results in fuzzy $b$-metric space using ordered-theoretic relation, which can also be seen in the work of Alam et al. [24].

Inspired by the metric structure used by Ali et al. [22] and using the concept of $m$-metric space, we introduce the notion of $\mathfrak{R}_{m}$-metric space. We improve and generalize some wellknown results and establish fixed point theorems in the sense of $\mathfrak{R}_{m}$-metric space. We also impart some illustrative examples and a possible application for Fredholm integral equations to demonstrate the validity of our results. 
Definition 1 ([2]). Let $Z$ be a nonempty set. A function $\rho: Z \times Z \longrightarrow[0,+\infty)$ is called a partial metric $(p m)$, if the following conditions are satisfied:

(1) $\omega=v$ iff $\rho(\omega, \omega)=\rho(\omega, v)=\rho(\nu, v)$;

(2) $\rho(\omega, \omega) \leq \rho(\omega, v)$, for all $\omega, v \in Z$;

(3) $\rho(\omega, v)=\rho(v, \omega)$, for all $\omega, v \in Z$,

(4) $\rho(\omega, v) \leq \rho(\omega, \mu)+\rho(\mu, v)-\rho(\mu, \mu)$, for all $\omega, v$, $\mu \in Z$.

The pair $(Z, \rho)$ is called a partial metric space (pms).

Notation 2 [11].

(1) $m_{\omega, v}=\min \{m(\omega, \omega), m(\nu, v)\}$

(2) $M_{\omega, v}=\max \{m(\omega, \omega), m(v, v)\}$ follows.

In [11], a generalization of the pms was introduced as

Definition 3 ([11]). Let $Z$ be a nonempty set. A function $m: Z \times Z \longrightarrow[0,+\infty)$ is called an $m$-metric, if the following conditions are satisfied:

(1) $\omega=v$ iff $m(\omega, \omega)=m(\omega, v)=m(v, v)$;

(2) $m_{\omega, v} \leq m(\omega, v)$, for all $\omega, v \in Z$;

(3) $m(\omega, v)=m(\nu, \omega)$, for all $\omega, v \in Z$;

(4) $\left(m(\omega, v)-m_{\omega, v}\right) \leq\left(m(\omega, \mu)-m_{\omega, \mu}\right)+(m(\mu, v)-$ $\left.m_{\mu, v}\right)$, for all $\omega, v, \mu \in Z$.

The pair $(Z, m)$ is called an $m$-ms.

Example 4. Let $Z=[0,+\infty)$. Then $m(\omega, v)=|\omega-v|$ on $Z$ is an m-metric.

Definition 5 ([25]). Let $Z$ be a nonempty set. A subset $\mathfrak{R}$ of $Z^{2}$ is called a binary relation on $Z$. Then, for any $\omega, v \in Z$, we say that " $\omega$ is $\mathfrak{R}$-related to $v$," that is, $\omega \Re v$, or " $\omega$ relates to $v$ under $\mathfrak{R}$ ” iff $(\omega, v) \in \mathfrak{R}$. $(\omega, v) \notin \mathfrak{R}$ means that “ $\omega$ is not $\mathfrak{R}$ -related to $v$ " or " $v$ is not related to $\omega$ under $\Re$."

Definition 6 ([25]). A binary relation $\mathfrak{R}$ defined on a nonempty set $\mathrm{Z}$ is called

(a) reflexive if $(\omega, \omega) \in \Re \forall \omega \in Z$;

(b) irreflexive if $(\nu, v) \notin \Re$ for some $v \in Z$;

(c) symmetric if $(\omega, v) \in \mathfrak{R}$ implies $(\nu, \omega) \in \mathfrak{R} \forall \omega, v \in Z$;

(d) antisymmetric if $(\omega, v) \in \mathfrak{R}$ and $(\nu, \omega) \in \mathfrak{R}$ imply $\omega=v \forall \omega, v \in Z$; (e) transitive if $(\omega, v) \in \Re$ and $(\nu, \mu) \in \Re$ imply $(\omega, \mu)$ $\in \Re \forall \omega, \nu, \mu \in Z$;

(f) preorder if $\mathfrak{R}$ is reflexive and transitive.

Definition 7 ([21]). Let $(Z, \mathfrak{R})$ be an $\mathfrak{R}$-set. A sequence $\left\{\omega_{i}\right\}$ is called an $\mathfrak{R}$-sequence $(\mathfrak{R} S)$ if

$$
\left(\forall i \in \mathbb{N}, \omega_{i} \mathfrak{R} \omega_{i+1}\right) \text { or }\left(\forall i \in \mathbb{N}, \omega_{i+1} \mathfrak{R} \omega_{i}\right)
$$

Definition 8 ([21]). A map $g: Z \longrightarrow Z$ is $\mathfrak{R}$-continuous(or $\Re C$ ) in $\omega \in Z$ if for each $\mathfrak{R} S\left\{\omega_{i}\right\}_{i \in \mathbb{N}}$ in $Z$ such that $\omega_{i} \longrightarrow \omega$ then $g\left(\omega_{i}\right) \longrightarrow g(\omega)$. Also, $g$ is said to be $\mathfrak{R C}$ on $Z$, if $g$ is $\mathfrak{R} C$ for each $\omega \in Z$.

Definition 9 ([18]). Let $(Z, d)$ be a ms and $\Re$ be a binary relation on $Z$. Then $(Z, d, \mathfrak{R})$ is called an $\mathfrak{R}$-ms.

Definition 10 ([18]). Let $(Z, \Re, d)$ be an $\Re$-ms. Then $Z$ is said to be $\mathfrak{R}$-complete, if every Cauchy $\mathfrak{R} S$ is convergent.

Definition 11 ([18]). Let $(Z, \mathfrak{R})$ be an $\mathfrak{R}$-set. A map $g: Z$ $\longrightarrow Z$ is said to be $\mathfrak{R}$-preserving $(\mathfrak{R} P)$ if $g \omega \Re \mathfrak{R} v$ whenever $\omega \Re v$. Also, $g: Z \longrightarrow Z$ is said to be weakly $\Re P$, if $g \omega \Re g v$ or $g \nu \mathfrak{R} g \omega$ whenever $\omega \mathfrak{R} v$.

Definition 12 [21]. A map $\mathrm{g}: \mathrm{Z} \longrightarrow \mathrm{Z}$ is an $\mathfrak{R}$-contraction, if

$$
d(g \omega, g v) \leq k d(\omega, v)
$$

for all $\omega, v \in Z$ with $\omega \Re v$, where $0<k<1$.

\section{Main Results}

We will start this section with the definition of an $\mathfrak{R}_{m}$-ms, but first, we introduce the following notations, which would be helpful during the proof.

Notation 13.
(1) $\sigma_{\Re \omega, v}=\min \left\{\sigma_{\Re}(\omega, \omega), \sigma_{\mathfrak{R}}(\nu, v)\right\}$;
(2) $\sigma_{\mathfrak{R} \omega, v}^{\prime}=\max \left\{\sigma_{\mathfrak{R}}(\omega, \omega), \sigma_{\mathfrak{R}}(\nu, v)\right\}$.

Definition 14 . Let $Z \neq \varnothing$ and $\Re$ be a reflexive binary relation (br) on $Z$, denoted as $(Z, \mathfrak{R})$. A map $\sigma_{\mathfrak{R}}: Z \times Z \longrightarrow \mathbb{R}^{+}$is called an $\mathfrak{R}_{m}$-metric on $Z$, if the following conditions are satisfied, for all $\omega, \nu, \mu \in Z$ with either $\omega \mathfrak{R} \nu$ or $\nu \Re \omega$, either $\omega \Re$ or $\mu \mathfrak{R} \omega$ and either $\mu \mathfrak{R} \nu$ or $\nu \mathfrak{R} \mu$ :

$\left(\sigma_{\mathfrak{R}} 1\right) \omega=\nu$ iff $\sigma_{\mathfrak{R}}(\omega, \omega)=\sigma_{\mathfrak{R}}(\omega, v)=\sigma_{\mathfrak{R}}(\nu, v)$;

$\left(\sigma_{\mathfrak{R}}^{2}\right) \sigma_{\mathfrak{R} \omega, v} \leq \sigma_{\mathfrak{R}}(\omega, v)$;

$\left(\sigma_{\mathfrak{R}} 3\right) \sigma_{\mathfrak{R}}(\omega, v)=\sigma_{\mathfrak{R}}(\nu, \omega)$;

$\left(\sigma_{\mathfrak{R}} 4\right)$

$\left(\sigma_{\mathfrak{R}}(\omega, v)-\sigma_{\mathfrak{R} \omega, \nu}\right) \leq\left(\sigma_{\mathfrak{R}}(\omega, \mu)-\sigma_{\mathfrak{R} \omega, \mu}\right)+\left(\sigma_{\mathfrak{R}}(\mu, v)-\sigma_{\mathfrak{R} \mu, v}\right)$.

Then, $\left(Z, \mathfrak{R}, \sigma_{\mathfrak{R}}\right)$ is called an $\mathfrak{R}_{m}-\mathrm{ms}$. 
Remark 15. In the above definition, a set $Z$ is endowed with a reflexive br $\Re$ and $\sigma_{\mathfrak{R}}: Z \times Z \longrightarrow \mathbb{R}^{+}$satisfies $\left(\sigma_{\mathfrak{R}} 1\right)-\left(\sigma_{\mathfrak{R}} 4\right)$ only for those elements which are comparable under the reflexive br $\mathfrak{R}$. Hence, the $\mathfrak{R}_{m}$-ms may not be a $m$-metric, but the converse is true.

The next example shows that the $\mathfrak{R}_{m}$-ms does not need to be an $m$-ms.

Example 16. Let $Z=\mathbb{R}$ and a br $\mathfrak{R}$ be defined by: $\omega \Re \mathcal{R}$ iff $\omega=v$ or $\omega, v>0$. It is easy to prove that $\sigma_{\Re}(\omega, v)=\max$ $\{|\omega|,|\nu|\}$ is an $\mathfrak{R}_{m}$-metric on $Z$, but $\sigma_{\mathfrak{R}}$ is not an $m$-metric on $Z$; since for $\omega=-2$ and $\nu=2$, we have $\sigma_{\mathfrak{R}}(\omega, \omega)=\sigma_{\mathfrak{R}}(\omega$, $v)=\sigma_{\mathfrak{R}}(v, v)=2$

Definition 17. Let $\left(Z, \mathfrak{R}, \sigma_{\mathfrak{R}}\right)$ be an $\mathfrak{R}_{m}$-ms. Let $\left\{\omega_{n}\right\}$ be an $\mathfrak{R} S$ in $\left(Z, \mathfrak{R}, \sigma_{\mathfrak{R}}\right)$, that is, $\omega_{n} \mathfrak{R} \omega_{n+1}$ or $\omega_{n+1} \mathfrak{R} \omega_{n}$ for each $n \in \mathbb{N}$. Then, $\left\{\omega_{n}\right\}$ is said to be

(i) $\mathfrak{R}$-convergent to some $\omega \in Z$, if

$$
\lim _{i \longrightarrow \infty}\left(\sigma_{\mathfrak{R}}\left(\omega_{i}, \omega\right)-\sigma_{\mathfrak{R} \omega_{i}, \omega}\right)=0
$$

(ii) $\mathfrak{R}$-Cauchy if

$$
\lim _{i, j \longrightarrow \infty}\left(\sigma_{\Re}\left(\omega_{i}, \omega_{j}\right)-\sigma_{\Re \omega_{i}, \omega_{j}}\right), \lim _{i, j \longrightarrow \infty}\left(\sigma_{\Re \omega_{i}, \omega_{j}}-\sigma_{\Re \omega_{i}, \omega_{j}}\right)
$$

exist and are finite.

Definition 18. (Z, $\left.\mathfrak{R}, \sigma_{\mathfrak{R}}\right)$ is said to be $\mathfrak{R}$-complete $\mathfrak{R}_{m}$-ms, if for every $\mathfrak{R}$-Cauchy sequence in $Z$ is convergent.

Definition 19. Let $\left(Z, \mathfrak{R}, \sigma_{\Re}\right)$ be an $\mathfrak{R}_{m}$-ms. We say that $g: Z \longrightarrow Z$ is $\mathfrak{R} C$ at $h \in Z$ if for each $\mathfrak{R} S\left\{\omega_{i}\right\}$ in $Z$ with $\sigma_{\mathfrak{R}}\left(\omega_{i}, \omega\right) \longrightarrow 0$, we have $\sigma_{\mathfrak{R}}\left(g \omega_{i}, g \omega\right) \longrightarrow 0$. Also, $g$ is $\mathfrak{R} C$ on $Z$ if $g$ is $\mathfrak{R} C$ for each $\omega \in Z$.

The following results help us to ensure the existence of fixed point (fp) for self-maps. Throughout, we assume that $\mathfrak{R}$ is a preorder relation.

Theorem 20. Let $\left(Z, \mathfrak{R}, \sigma_{\mathfrak{R}}\right)$ be an $\mathfrak{R}$-complete $\mathfrak{R}_{m}-m s$ and $g: Z \longrightarrow Z$ be $\mathfrak{R} P$ and $\mathfrak{R}$ satisfying the following condition:

$$
\sigma_{\Re}(g \omega, g v) \leq z \sigma_{\Re}(\omega, v), \text { for all } \omega, v \in Z \text { with } \omega \Re \text {, }
$$

where $z \in[0,1)$. Then, g has a uniquefp $\omega \in Z$ and $\sigma_{\mathfrak{R}}(\omega, \omega)=0$.

Proof. Let $\omega_{1}=g \omega_{0}, \omega_{2}=g \omega_{1}, \omega_{3}=g \omega_{2}, \omega_{4}=g \omega_{3}, \cdots, \omega_{i+1}$ $=g \omega_{i}$, for all $i \in \mathbb{N}$. Since $g$ is $\mathfrak{R} P,\left\{\omega_{i}\right\}$ is $\mathfrak{R} S$. Then, by (5), we get

$$
\sigma_{\Re}\left(\omega_{i}, \omega_{i+1}\right)=\sigma_{\Re}\left(g \omega_{i-1}, g \omega_{i}\right) \leq z^{i} \sigma_{\mathfrak{R}}\left(\omega_{0}, \omega_{1}\right),
$$

for all $i \in \mathbb{N}$. For any $i, j \in \mathbb{N}$ and $i<j$, it follows that

$$
\begin{aligned}
\sigma_{\mathfrak{R}}\left(\omega_{i}, \omega_{j}\right) & =\sigma_{\mathfrak{R}}\left(g \omega_{i-1}, g \omega_{j-1}\right) \leq z \sigma_{\mathfrak{R}}\left(\omega_{i-1}, \omega_{j-1}\right) \\
& \leq z \sigma_{\mathfrak{R}}\left(z \sigma_{\mathfrak{R}}\left(\omega_{i-2}, \omega_{j-2}\right)\right) \\
& \leq z^{2} \sigma_{\mathfrak{R}}\left(\omega_{i-2}, \omega_{j-2}\right) \leq \cdots \leq z^{i} \sigma_{\mathfrak{R}}\left(\omega_{1}, \omega_{j-i}\right) .
\end{aligned}
$$

Hence,

$$
\begin{aligned}
\sigma_{\mathfrak{R}}\left(\omega_{i}, \omega_{j}\right)-\sigma_{\mathfrak{R} \omega_{i}, \omega_{j}} & \leq z^{i}\left(\sigma_{\mathfrak{R}}\left(\omega_{0}, \omega_{1}\right)+\sigma_{\mathfrak{R}}\left(\omega_{1}, \omega_{j-i}\right)\right) \\
& \leq z^{i}\left(\sigma_{\mathfrak{R}}\left(\omega_{0}, \omega_{1}\right)+\sigma_{\mathfrak{R}}\left(\omega_{1}, \omega_{2}\right)+\sigma_{\mathfrak{R}}\left(\omega_{2}, \omega_{j-i}\right)\right) \\
& \leq z^{i}\left(\sigma_{\mathfrak{R}}\left(\omega_{0}, \omega_{1}\right)+\sigma_{\mathfrak{R}}\left(\omega_{1}, \omega_{2}\right)+\cdots+\sigma_{\mathfrak{R}}\left(\omega_{j-i-1}, \omega_{j-i}\right)\right) \\
& \leq z^{i}\left(\sigma_{\mathfrak{R}}\left(\omega_{0}, \omega_{1}\right)+z \sigma_{\mathfrak{R}}\left(\omega_{0}, \omega_{1}\right)+\cdots+z^{j-1} \sigma_{\mathfrak{R}}\left(\omega_{0}, \omega_{1}\right)\right) \\
& \leq z^{i}\left(1+z+z^{2}+\cdots+z^{j-1}\right) \sigma_{\mathfrak{R}}\left(\omega_{0}, \omega_{1}\right) \\
& \leq \frac{z^{i}}{1-z} \sigma_{\mathfrak{R}}\left(\omega_{0}, \omega_{1}\right) .
\end{aligned}
$$

As $z \in[0,1)$, it follows from the above inequality that

$$
\sigma_{\Re}\left(\omega_{i}, \omega_{j}\right)-\sigma_{\Re \omega_{i}, \omega_{j}} \longrightarrow 0 \text { as } i, j \longrightarrow \infty \text {. }
$$

Similarly,

$$
\sigma_{\mathfrak{R} \omega_{i}, \omega_{j}}{ }^{\prime}-\sigma_{\mathfrak{R} \omega_{i}, \omega_{j}} \longrightarrow 0 \text { as } i, j \longrightarrow \infty .
$$

Thus, $\left\{\omega_{i}\right\}$ is a $\mathfrak{R}$-Cauchy in $Z$. Since $Z$ is $\mathfrak{R}$-complete, there exists $\omega \in Z$ such that

$$
\sigma_{\Re}\left(\omega_{i}, \omega\right)-\sigma_{\Re \omega_{i}, \omega} \longrightarrow 0 \text { as } i \longrightarrow \infty
$$

Now, we show that $\omega$ is a fp of $g$. Consider

$$
\begin{aligned}
\sigma_{\mathfrak{R}}(\omega, g \omega) & \leq \limsup _{i \longrightarrow \infty} \sigma_{\mathfrak{R}}\left(\omega, \omega_{i}\right)+\underset{i \longrightarrow \infty}{\limsup } \sigma_{\mathfrak{R}}\left(\omega_{i}, g \omega\right) \\
& =\limsup _{i \longrightarrow \infty} \sigma_{\mathfrak{R}}\left(\omega_{i}, g \omega\right) \\
& =\limsup _{i \longrightarrow \infty} \sigma_{\mathfrak{R}}\left(g \omega_{i-1}, g \omega\right) \\
& \leq \limsup _{i \longrightarrow \infty} z \sigma_{\mathfrak{R}}\left(\omega_{i-1}, \omega\right) .
\end{aligned}
$$

By using $\left(\sigma_{\mathfrak{R}} 4\right)$, we get

$$
\begin{aligned}
\sigma_{\mathfrak{R}}(\omega, g \omega) \leq & \underset{i \longrightarrow \infty}{\limsup } z\left[\left(\sigma_{\mathfrak{R}}\left(\omega_{i-1}, g \omega\right)-\sigma_{\mathfrak{R} \omega_{i-1}, g \omega}\right)\right. \\
& \left.+\left(\sigma_{\mathfrak{R}}(g \omega, \omega)-\sigma_{\mathfrak{R} g \omega \omega}\right)\right]-\sigma_{\mathfrak{R}}(g \omega, g \omega) \\
\leq & z \sigma_{\mathfrak{R}}(g \omega, \omega)
\end{aligned}
$$

implies

$$
\begin{array}{r}
\sigma_{\mathfrak{R}}(\omega, g \omega)=0 \\
0 \leq \sigma_{\mathfrak{R}}(g \omega, g \omega) \leq z \sigma_{\mathfrak{R}}(\omega, \omega)=0 .
\end{array}
$$


Hence,

$$
\sigma_{\mathfrak{R}}(g \omega, g \omega)=\sigma_{\mathfrak{R}}(\omega, g \omega)=\sigma_{\mathfrak{R}}(\omega, \omega) .
$$

By $\left(\sigma_{\mathfrak{R}} 1\right)$, we get $g \omega=\omega$ as desired. Then,

Now, we show that if $\omega$ is a fp of $g$, then $\sigma_{\mathfrak{R}}(\omega, \omega)=0$.

$$
\sigma_{\mathfrak{R}}(\omega, \omega)=\sigma_{\mathfrak{R}}\left(g^{i} \omega, g^{i} \omega\right) \leq z \sigma_{\mathfrak{R}}(\omega, \omega)<\sigma_{\mathfrak{R}}(\omega, \omega),
$$

and hence, $\sigma_{\mathfrak{R}}(\omega, \omega)=0$.

Finally, we show that fixed point of $g$ is unique. Assume that there exists $v \in Z$ such that $g v=v$. Hence, we have $g^{i} \omega=\omega, g^{i} v=v$ for all $i \in \mathbb{N}$. By the choice of $\omega_{0}$ in the first part of the proof, we obtain

$$
\left[\omega_{0} \mathfrak{R} \omega \text { and } \omega_{0} \mathfrak{R} v\right] \text { or }\left[\omega \Re \omega_{0} \text { and } \nu \Re \omega_{0}\right] .
$$

Since $g$ is $\mathfrak{R} P$, we have

$$
\left[g^{i} \omega_{0} \Re g^{i} \omega \text { and } g^{i} \omega_{0} \Re g^{i} v\right] \text {, }
$$

or

$$
\left[g^{i} \omega \Re g^{i} \omega_{0} \text { and } g^{i} v \Re g^{i} \omega_{0}\right],
$$

for all $i \in \mathbb{N}$. Thus,

$$
\sigma_{\mathfrak{R}}(\omega, v)=\sigma_{\mathfrak{R}}\left(g^{i} \omega, g^{i} v\right) \leq z \sigma_{\mathfrak{R}}(\omega, v)<\sigma_{\mathfrak{R}}(\omega, v),
$$

which implies that $\sigma_{\mathfrak{R}}(\omega, v)=0$ and so $\sigma_{\mathfrak{R}}(\omega, \omega)=\sigma_{\mathfrak{R}}(\omega$, $v)=\sigma_{\mathfrak{R}}(v, v)$. Thus, $\omega=v$.

Example 21. Let $Z=\mathbb{R}$, and $\sigma_{\mathfrak{R}}: Z \times Z \longrightarrow \mathbb{R}^{+}$be given by

$$
\sigma_{\mathfrak{R}}(\omega, v)= \begin{cases}|\omega-v|^{2}, & \text { if } \omega, v \geq 0 \\ 0, & \text { otherwise }\end{cases}
$$

Define the binary relation $\Re$ on $Z$ by $\omega \Re v$ if $\omega=v$ or $\omega, v \geq 0$. Then $\left(Z, \mathfrak{R}, \sigma_{\mathfrak{R}}\right)$ is $\mathfrak{R}$-complete $\mathfrak{R}_{m}$-ms. Define $g: Z \longrightarrow Z$ by

$$
g(\omega)=\left\{\begin{array}{cc}
\frac{\omega}{3}, & 0 \leq \omega \leq 3, \\
0, & \text { otherwise. }
\end{array}\right.
$$

Then, it is very simple to verify the following:

If $\omega=v$, then $g \omega=g v$. If $\omega, v \geq 0$, then $g \omega, g v \geq 0$. Thus, $g$ is $\mathfrak{R} P$.

Also, one can see that $|g(\omega)-g(v)|^{2} \leq 1 / 9|\omega-v|^{2}$ for all $\omega, v \in Z$ with $\omega \Re v$. Hence, the inequality (5) is satisfied with $z=1 / 9$. But $g$ is not a contraction. Otherwise, for two points $11 / 4$ and $20 / 4$ and for all $0<c<1 / 3$, we have $|g(11 / 4)-g(20 / 4)|^{2} \leq c|11 / 4-20 / 4|^{2}$ and one can conclude that it is a contradiction.

Let $\left\{\omega_{i}\right\}$ be an arbitrary $\Re S$ in $Z$ such that $\left\{\omega_{i}\right\}$ converges to $\omega \in Z$. Since the inequality (5) is satisfied, for each $i \in \mathbb{N}$, we have

$$
\left|g\left(\omega_{i}\right)-g(\omega)\right|^{2} \leq \frac{1}{9}\left|\omega_{i}-\omega\right|^{2} .
$$

As $i$ goes to infinity, $g$ is $\Re C$. But it can be easily seen that $g$ is not continuous. Therefore, all the conditions of Theorem 20 are satisfied. Hence, we can conclude that $g$ has a unique fixed point in $Z$ which is 0 .

Remark 22. Note that the function $\sigma_{\mathfrak{R}}$ defined in the above example is neither a metric, nor an $m$-metric on $\mathbb{R}$. Since $\sigma_{\mathfrak{R}}$ $(4,1)=9, \sigma_{\mathfrak{R}}(4,-1)=0, \sigma_{\mathfrak{R}}(-1,1)=0$, and $\sigma_{\mathfrak{R}}(-1,-1)=0$.

Theorem 23. Let $\left(Z, \Re, \sigma_{\mathfrak{R}}\right)$ be an $\mathfrak{R}$-complete $\mathfrak{R}_{m}$-ms and $g: Z \longrightarrow Z$ be $\Re P$ and $\Re C$ satisfying the following condition:

$$
\sigma_{\mathfrak{R}}(g \omega, g v) \leq z\left[\sigma_{\mathfrak{R}}(\omega, g \omega)+\sigma_{\mathfrak{R}}(v, g v)\right]
$$

for all $\omega, v \in Z$ with $\omega \Re v$, where $z \in[0,1)$. Then $g$ has a unique $f p \omega \in Z$ and $\sigma_{\mathfrak{R}}(\omega, \omega)=0$.

Proof. Let $\omega_{1}=g \omega_{0}, \omega_{2}=g \omega_{1}, \omega_{3}=g \omega_{2}, \omega_{4}=g \omega_{3}, \cdots, \omega_{i+1}=g$ $\omega_{i}$, for all $i \in \mathbb{N}$. Since $g$ is $\Re P$, then $\left\{\omega_{i}\right\}$ is $\mathfrak{R S}$. Then by (24), we get

$$
\begin{aligned}
\sigma_{\mathfrak{R}}\left(\omega_{i}, \omega_{i+1}\right) & =\sigma_{\mathfrak{R}}\left(g \omega_{i-1}, g \omega_{i}\right) \\
& \leq z\left[\sigma_{\mathfrak{R}}\left(\omega_{i-1}, g \omega_{i-1}\right)+\sigma_{\mathfrak{R}}\left(\omega_{i}, g \omega_{i}\right)\right] \\
& =z\left[\sigma_{\mathfrak{R}}\left(\omega_{i-1}, \omega_{i}\right)+\sigma_{\mathfrak{R}}\left(\omega_{i}, \omega_{i+1}\right)\right],
\end{aligned}
$$

for $\quad$ all $\quad i \in \mathbb{N} . \sigma_{\mathfrak{R}}\left(\omega_{i}, \omega_{i+1}\right) \leq z \sigma_{\mathfrak{R}}\left(\omega_{i-1}, \omega_{i}\right)+z \sigma_{\mathfrak{R}}\left(\omega_{i}, \omega_{i+1}\right)$ which implies $\sigma_{\mathfrak{R}}\left(\omega_{i}, \omega_{i+1}\right) \leq c \sigma_{\mathfrak{R}}\left(\omega_{i-1}, \omega_{i}\right)$, where $c=z / 1-$ $z<1$ as $z \in[0,1)$. By repeating this process,

$$
\sigma_{\mathfrak{R}}\left(\omega_{i}, \omega_{i+1}\right) \leq c^{i} \sigma_{\mathfrak{R}}\left(\omega_{0}, \omega_{1}\right)
$$

Then $\lim _{i \rightarrow \infty} \sigma_{\mathfrak{R}}\left(\omega_{i}, \omega_{i+1}\right)=0$. By (24), for any two natural number $j>i$, it follows that

$$
\begin{aligned}
\sigma_{\mathfrak{R}}\left(\omega_{i}, \omega_{j}\right) & \leq \sigma_{\mathfrak{R}}\left(g \omega_{i-1}, g \omega_{j-1}\right) \\
& \leq z\left[\sigma_{\mathfrak{R}}\left(\omega_{i-1}, g \omega_{i-1}\right)+\sigma_{\mathfrak{R}}\left(\omega_{j-1}, g \omega_{j-1}\right)\right] \\
& =z\left[\sigma_{\mathfrak{R}}\left(\omega_{i-1}, \omega_{i}\right)+\sigma_{\mathfrak{R}}\left(\omega_{j-1}, \omega_{j}\right)\right],
\end{aligned}
$$

and so $\lim _{i \longrightarrow \infty} \sigma_{\mathfrak{R}}\left(\omega_{i}, \omega_{j}\right)=0$. For every $\xi>0$, we can find a natural number $i_{0}$ such that $\sigma_{\mathfrak{R}}\left(\omega_{i}, \omega_{i+1}\right)<\xi / 2$ and $\sigma_{\mathfrak{R}}\left(\omega_{j}\right.$, $\left.\omega_{j+1}\right)<\xi / 2$, for all $i, j>i_{0}$. Therefore, it follows that

$$
\begin{aligned}
\sigma_{\mathfrak{R}}\left(\omega_{i}, \omega_{j}\right) & \leq z\left[\sigma_{\mathfrak{R}}\left(\omega_{i-1}, \omega_{i}\right)+\sigma_{\mathfrak{R}}\left(\omega_{j-1}, \omega_{j}\right)\right] \\
& <z\left[\frac{\xi}{2}+\frac{\xi}{2}\right]<\frac{\xi}{2}+\frac{\xi}{2}=\xi
\end{aligned}
$$

for all $i, j>i_{0}$, and $\sigma_{\mathfrak{R}}\left(\omega_{i}, \omega_{j}\right) \longrightarrow 0$ as $i, j \longrightarrow \infty$

$$
\sigma_{\mathfrak{R}}\left(\omega_{i}, \omega_{j}\right)-\sigma_{\mathfrak{R} \omega_{i}, \omega_{j}}<\xi \text {, for all } i, j>i_{0} .
$$


Now,

$$
\left[g^{i} \omega_{0} \Re g^{i} \omega \text { and } g^{i} \omega_{0} \Re g^{i} v\right] \text {, }
$$

$$
\begin{aligned}
\sigma_{\mathfrak{R}}\left(\omega_{i}, \omega_{i}\right) & =\sigma_{\mathfrak{R}}\left(g \omega_{i-1}, g \omega_{i-1}\right) \\
& \leq z\left[\sigma_{\mathfrak{R}}\left(\omega_{i-1}, g \omega_{i-1}\right)+\sigma_{\mathfrak{R}}\left(\omega_{i-1}, g \omega_{i-1}\right)\right] \\
& =z\left[\sigma_{\mathfrak{R}}\left(\omega_{i-1}, \omega_{i}\right)+\sigma_{\mathfrak{R}}\left(\omega_{i-1}, \omega_{i}\right)\right] \\
& \leq 2 z \sigma_{\mathfrak{R}}\left(\omega_{i-1}, \omega_{i}\right) \longrightarrow 0 \text { as } i \longrightarrow \infty
\end{aligned}
$$

So $\sigma_{\mathfrak{R}}\left(\omega_{i}, \omega_{i}\right) \longrightarrow 0$ which implies that

$$
\sigma_{\mathfrak{R}}\left(\omega_{i}, \omega_{j}\right)-\sigma_{\mathfrak{R} \omega_{i}, \omega_{j}} \longrightarrow 0 \text { as } i, j \longrightarrow \infty .
$$

Similarly,

$$
\sigma_{\mathfrak{R} \omega_{i}, \omega_{j}}{ }^{\prime}-\sigma_{\mathfrak{R} \omega_{i}, \omega_{j}} \longrightarrow 0 \text { as } i, j \longrightarrow \infty
$$

Thus, $\left\{\omega_{i}\right\}$ is a $\mathfrak{R}$-Cauchy in $Z$. Since $Z$ is $\mathfrak{R}$-complete, there exists $\omega \in Z$ such that

$$
\sigma_{\mathfrak{R}}\left(\omega_{i}, \omega\right)-\sigma_{\mathfrak{R} \omega_{i}, \omega} \longrightarrow 0 \text { as } i \longrightarrow \infty .
$$

Now, we show that $\omega$ is a fp of $g$. Consider,

$$
\begin{aligned}
\sigma_{\mathfrak{R}}(\omega, g \omega) & \leq \limsup _{i \longrightarrow \infty} \sigma_{\mathfrak{R}}\left(\omega, \omega_{i}\right)+\underset{i \longrightarrow \infty}{\limsup } \sigma_{\mathfrak{R}}\left(\omega_{i}, g \omega\right) \\
& =\limsup _{n \longrightarrow \infty} \sigma_{\mathfrak{R}}\left(\omega_{i}, g \omega\right) \\
& \leq \limsup _{i \longrightarrow \infty}\left[z\left(\sigma_{\mathfrak{R}}\left(\omega_{i-1}, g \omega_{i-1}\right)+\sigma_{\mathfrak{R}}(\omega, g \omega)\right)\right] \\
& \leq \limsup _{i \longrightarrow \infty} z \sigma_{\mathfrak{R}}\left(\omega_{i-1}, \omega_{i}\right)+\underset{i \longrightarrow \infty}{\limsup } z \sigma_{\mathfrak{R}}(\omega, g \omega) \\
& \leq z \sigma_{\mathfrak{R}}(\omega, g \omega),
\end{aligned}
$$

implies

$$
\begin{aligned}
\sigma_{\mathfrak{R}}(\omega, g \omega) & =0 \\
0 \leq \sigma_{\mathfrak{R}}(g \omega, g \omega) \leq 2 z \sigma_{\mathfrak{R}}(\omega, \omega) & =0 .
\end{aligned}
$$

Hence,

$$
\sigma_{\mathfrak{R}}(g \omega, g \omega)=\sigma_{\mathfrak{R}}(\omega, g \omega)=\sigma_{\mathfrak{R}}(\omega, \omega) .
$$

By $\left(\sigma_{\mathfrak{R}} 1\right)$, we get $g \omega=\omega$.

Now, we show that if $\omega$ is a fp of $g$, then $\sigma_{\mathfrak{R}}(\omega, \omega)=0$. Consider,

$$
\begin{aligned}
\sigma_{\mathfrak{R}}(\omega, \omega) & =\sigma_{\mathfrak{R}}\left(g^{i} \omega, g^{i} \omega\right) \leq z\left[\sigma_{\mathfrak{R}}(\omega, g \omega)+\sigma_{\mathfrak{R}}(\omega, g \omega)\right] \\
& =2 z \sigma_{\mathfrak{R}}(\omega, g \omega)=2 z \sigma_{\mathfrak{R}}(\omega, \omega),
\end{aligned}
$$

that is, $\sigma_{\mathfrak{R}}(\omega, \omega)=0$.

Finally, we show that fixed point of $g$ is a unique. Assume that there exists $v \in Z$ such that $g v=v$. Since $g$ is $\Re P$, we have or

$$
\left[g^{i} \omega \Re g^{i} \omega_{0} \text { and } g^{i} \nu \Re g^{i} \omega_{0}\right]
$$

for all $i \in \mathbb{N}$. Thus,

$$
\begin{aligned}
\sigma_{\mathfrak{R}}(\omega, v) & =\sigma_{\mathfrak{R}}\left(g^{i} \omega, g^{i} v\right) \leq z\left[\sigma_{\mathfrak{R}}(\omega, g \omega)+\sigma_{\mathfrak{R}}(v, g v)\right] \\
& =z\left[\sigma_{\mathfrak{R}}(\omega, \omega)+\sigma_{\mathfrak{R}}(v, v)\right]=0
\end{aligned}
$$

which implies that $\sigma_{\mathfrak{R}}(\omega, v)=0$ and so $\sigma_{\mathfrak{R}}(\omega, \omega)=\sigma_{\mathfrak{R}}(\omega$, $v)=\sigma_{\mathfrak{R}}(\nu, v)$. Thus, $\omega=v$.

Theorem 24. Let $\left(Z, \mathfrak{R}, \sigma_{\mathfrak{R}}\right)$ be an $\mathfrak{R}$-complete $\mathfrak{R}_{m}$-ms and $g: Z \longrightarrow Z$ be $\Re P$ and $\Re C$ satisfying the following condition:

$$
\sigma_{\mathfrak{R}}(g \omega, g v) \leq z \max \left\{\sigma_{\mathfrak{R}}(\omega, v), \sigma_{\mathfrak{R}}(\omega, g \omega), \sigma_{\mathfrak{R}}(v, g v)\right\}
$$

for all $\omega, v \in Z$ with $\omega \Re v$, where $z \in[0,1)$. Then, $g$ has a unique fp $\omega \in Z$ and $\sigma_{\mathfrak{R}}(\omega, \omega)=0$.

Proof. Let $\omega_{1}=g \omega_{0}, \omega_{2}=g \omega_{1}, \cdots, \omega_{i+1}=g \omega_{i}$, for all $i \in \mathbb{N}$. Since $g$ is $\mathfrak{R} P$, then $\left\{\omega_{i}\right\}$ is $\mathfrak{R} S$. By (41), we get

$$
\begin{aligned}
\sigma_{\mathfrak{R}}\left(\omega_{i+1}, \omega_{i}\right) & =\sigma_{\mathfrak{R}}\left(g \omega_{i}, g \omega_{i-1}\right) \\
& \leq z \max \left\{\sigma_{\mathfrak{R}}\left(\omega_{i}, \omega_{i-1}\right), \sigma_{\mathfrak{R}}\left(\omega_{i}, g \omega_{i}\right), \sigma_{\mathfrak{R}}\left(\omega_{i-1}, g \omega_{i-1}\right)\right\} \\
& =z \max \left\{\sigma_{\mathfrak{R}}\left(\omega_{i}, \omega_{i-1}\right), \sigma_{\mathfrak{R}}\left(\omega_{i}, \omega_{i+1}\right), \sigma_{\mathfrak{R}}\left(\omega_{i-1}, \omega_{i}\right)\right\} \\
& =z \max \left\{\sigma_{\mathfrak{R}}\left(\omega_{i}, \omega_{i-1}\right), \sigma_{\mathfrak{R}}\left(\omega_{i}, \omega_{i+1}\right)\right\}
\end{aligned}
$$

If $\max \left\{\sigma_{\mathfrak{R}}\left(\omega_{i}, \omega_{i-1}\right), \sigma_{\mathfrak{R}}\left(\omega_{i}, \omega_{i+1}\right)\right\}=\sigma_{\mathfrak{R}}\left(\omega_{i+1}, \omega_{i}\right)$. Then, from the above inequality, we obtain that $\sigma_{\mathfrak{R}}\left(\omega_{i+1}, \omega_{i}\right) \leq z$ $\sigma_{\mathfrak{R}}\left(\omega_{i+1}, \omega_{i}\right)$, a contradiction. Hence, $\max \left\{\sigma_{\mathfrak{R}}\left(\omega_{i}, \omega_{i-1}\right)\right.$, $\left.\sigma_{\mathfrak{R}}\left(\omega_{i}, \omega_{i+1}\right)\right\}=\sigma_{\mathfrak{R}}\left(\omega_{i}, \omega_{i-1}\right)$. From the above inequality, we have

$$
\sigma_{\mathfrak{R}}\left(\omega_{i+1}, \omega_{i}\right) \leq z \sigma_{\mathfrak{R}}\left(\omega_{i}, \omega_{i-1}\right)
$$

On repeating this process, we obtain

$$
\sigma_{\mathfrak{R}}\left(\omega_{i+1}, \omega_{i}\right) \leq z^{i} \sigma_{\mathfrak{R}}\left(\omega_{1}, \omega_{0}\right),
$$

for all $i \geq 0$. 


$$
\begin{aligned}
\sigma_{\mathfrak{R}}\left(\omega_{i}, \omega_{j}\right)-\sigma_{\mathfrak{R} \omega_{i}, \omega_{j}} & \leq z^{i}\left(\sigma_{\mathfrak{R}}\left(\omega_{0}, \omega_{1}\right)+\sigma_{\mathfrak{R}}\left(\omega_{1}, \omega_{j-i}\right)\right) \\
& \leq z^{i}\left(\sigma_{\mathfrak{R}}\left(\omega_{0}, \omega_{1}\right)+\sigma_{\mathfrak{R}}\left(\omega_{1}, \omega_{2}\right)+\sigma_{\mathfrak{R}}\left(\omega_{2}, \omega_{j-i}\right)\right) \\
& \leq z^{i}\left(\sigma_{\mathfrak{R}}\left(\omega_{0}, \omega_{1}\right)+\sigma_{\mathfrak{R}}\left(\omega_{1}, \omega_{2}\right)+\cdots+\sigma_{\mathfrak{R}}\left(\omega_{j-i-1}, \omega_{j-i}\right)\right) \\
& \leq z^{i}\left(\sigma_{\mathfrak{R}}\left(\omega_{0}, \omega_{1}\right)+z \sigma_{\mathfrak{R}}\left(\omega_{0}, \omega_{1}\right)+\cdots+z^{j-1} \sigma_{\mathfrak{R}}\left(\omega_{0}, \omega_{1}\right)\right) \\
& \leq z^{i}\left(1+z+z^{2}+\cdots+z^{j-1}\right) \sigma_{\mathfrak{R}}\left(\omega_{0}, \omega_{1}\right) \\
& \leq \frac{z^{i}}{1-z} \sigma_{\mathfrak{R}}\left(\omega_{0}, \omega_{1}\right) .
\end{aligned}
$$

As $z \in[0,1)$, it follows from the above inequality that

$$
\sigma_{\Re}\left(\omega_{i}, \omega_{j}\right)-\sigma_{\Re \omega_{i}, \omega_{j}} \longrightarrow 0 \text { as } i, j \longrightarrow \infty .
$$

Similarly,

$$
\sigma_{\Re}^{\prime}\left(\omega_{i}, \omega_{j}\right)-\sigma_{\Re \omega_{i}, \omega_{j}} \longrightarrow 0 \text { as } i, j \longrightarrow \infty
$$

Thus, $\left\{\omega_{i}\right\}$ is a $\mathfrak{R}$-Cauchy in $Z$. Since $Z$ is $\mathfrak{R}$-complete, there exists $\omega \in Z$ such that

$$
\sigma_{\mathfrak{R}}\left(\omega_{i}, \omega\right)-\sigma_{\mathfrak{R} \omega_{i}, \omega} \longrightarrow 0 \text { as } i \longrightarrow \infty
$$

Now, we show that $\omega$ is a fp of $g$. Consider

$$
\begin{aligned}
& \sigma_{\mathfrak{R}}(\omega, g \omega) \leq \limsup _{i \longrightarrow \infty} \sigma_{\mathfrak{R}}\left(\omega, \omega_{i}\right)+\underset{i \longrightarrow \infty}{\limsup } \sigma_{\mathfrak{R}}\left(\omega_{i}, g \omega\right) \\
& =\limsup \sigma_{\mathfrak{R}}\left(\omega_{i}, g \omega\right) \\
& =\limsup \sigma_{\mathfrak{R}}\left(g \omega_{i-1}, g \omega\right) \\
& \leq \limsup \left[z \max \left\{\sigma_{\Re}\left(\omega_{i-1}, \omega\right), \sigma_{\Re}\left(\omega_{i-1}, g \omega_{i-1}\right), \sigma_{\mathfrak{R}}(\omega, g \omega)\right\}\right] \\
& \leq z \max \left\{\limsup _{i \longrightarrow \infty} \sigma_{\mathfrak{R}}\left(\omega_{i-1}, \omega\right), \underset{i \longrightarrow \infty}{\limsup }\left(\omega_{i-1}, \omega_{i}\right), \limsup _{i \longrightarrow \infty} \sigma_{\mathfrak{R}}(\omega, g \omega)\right\} \\
& \leq z \sigma_{\Re}(\omega, g \omega) \text {. }
\end{aligned}
$$

So,

$$
\begin{aligned}
\sigma_{\mathfrak{R}}(\omega, g \omega)=0 \\
0 \leq \sigma_{\mathfrak{R}}(g \omega, g \omega) \\
\quad \leq z \max \left\{\sigma_{\mathfrak{R}}(\omega, \omega), \sigma_{\mathfrak{R}}(\omega, g \omega), \sigma_{\mathfrak{R}}(\omega, g \omega)\right\} \\
=\sigma_{\mathfrak{R}}(\omega, \omega) \\
=0 .
\end{aligned}
$$

Thus,

$$
\sigma_{\mathfrak{R}}(g \omega, g \omega)=\sigma_{\mathfrak{R}}(\omega, g \omega)=\sigma_{\mathfrak{R}}(\omega, \omega)
$$

By $\left(\sigma_{\mathfrak{R}} 1\right)$, we get $g \omega=\omega$ as desired.
Now, we show that if $\omega$ is a fp of $g$, then $\sigma_{\mathfrak{R}}(\omega, \omega)=0$. Then,

$$
\begin{aligned}
\sigma_{\mathfrak{R}}(\omega, \omega) & =\sigma_{\mathfrak{R}}\left(g^{i} \omega, g^{i} \omega\right) \\
& \leq z \max \left\{\sigma_{\mathfrak{R}}(\omega, \omega), \sigma_{\mathfrak{R}}(\omega, g \omega), \sigma_{\mathfrak{R}}(\omega, g \omega)\right\} \\
& <\sigma_{\mathfrak{R}}(\omega, \omega)
\end{aligned}
$$

and hence, $\sigma_{\mathfrak{R}}(\omega, \omega)=0$.

Finally, we show that fixed point of $g$ is a unique. Assume that there exists $v \in Z$ such that $g v=v$. Hence, we have $g^{i} \omega=\omega, g^{i} v=v$ for all $i \in \mathbb{N}$. By the choice of $\omega_{0}$ in the first part of the proof, we obtain

$$
\left[\omega_{0} \mathfrak{R} \omega \text { and } \omega_{0} \mathfrak{R} v\right] \text { or }\left[\omega \mathfrak{R} \omega_{0} \text { and } \nu \mathfrak{R} \omega_{0}\right]
$$

Since $g$ is $\mathfrak{R} P$, we have

$$
\left[g^{i} \omega_{0} \mathfrak{R} g^{i} \omega \text { and } g^{i} \omega_{0} \mathfrak{R} g^{i} v\right]
$$

or

$$
\left[g^{i} \omega \Re g^{i} \omega_{0} \text { and } g^{i} v \Re g^{i} \omega_{0}\right]
$$

for all $i \in \mathbb{N}$. Thus,

$$
\begin{aligned}
\sigma_{\mathfrak{R}}(\omega, v) & =\sigma_{\mathfrak{R}}\left(g^{i} \omega, g^{i} v\right) \\
& \leq z \max \left\{\sigma_{\mathfrak{R}}(\omega, v), \sigma_{\mathfrak{R}}(\omega, g \omega), \sigma_{\mathfrak{R}}(v, g v)\right\} \\
& =z \max \left\{\sigma_{\mathfrak{R}}(\omega, v), \sigma_{\mathfrak{R}}(\omega, \omega), \sigma_{\mathfrak{R}}(v, v)\right\} \\
& =z \sigma_{\mathfrak{R}}(\omega, v) \\
& <\sigma_{\mathfrak{R}}(\omega, v)
\end{aligned}
$$

which is a contradiction. Hence, $\sigma_{\Re}(\omega, v)=0$ and so $\sigma_{\mathfrak{R}}(\omega, \omega)=\sigma_{\mathfrak{R}}(\omega, v)=\sigma_{\mathfrak{R}}(\nu, v)$. Thus, $\omega=v$.

\section{Application}

Within this section, we are attempting to apply Theorem 20 to investigate the presence and uniqueness of solution for a Fredholm integral equation. The space of all continuous real valued functions defined on $I=[0,1]$ is considered to be $Z=C(I, \mathbb{R})$ with the $\mathfrak{R}_{m}$-metric given by

$$
\sigma_{\Re}(\omega(r), v(r))=\sup _{r \in I}\left|\frac{\omega(r)+v(r)}{2}\right|,
$$

for all $\omega, v \in Z$. It can also be equipped with a relation given by $\omega, \nu \in Z, \omega \mathfrak{R} v \Leftrightarrow \omega \leq \nu$. Then, $\left(Z, \mathfrak{R}, \sigma_{\mathfrak{R}}\right)$ is $\mathfrak{R}$-complete $\mathfrak{R}_{m}$-ms.

Consider the following Fredholm integral equation:

$$
\omega(r)=\int_{0}^{1} J(r, s, \omega(r)) d s, \quad r, s \in I,
$$

where $J \in C(I \times I \times \Re, \Re)$. 
Theorem 25. If there exists $z \in 0,1)$, such that

$$
|J(r, s, \omega(r))+J(r, s, v(r))| \leq z|\omega(r)+v(r)|, \quad r, s \in I,
$$

for all $\omega, v \in Z$. Then the integral equation (58) has a unique solution.

Proof. Define $g: Z \longrightarrow Z$ by

$$
g \omega(r)=\int_{0}^{1} J(r, s, \omega(r)) d s, \quad r, s \in I
$$

Observe that the presence of fixed point of an operator $g$ is identical to that of solution of the integral equation (58). Now, for all $\omega, v \in Z$ with $\omega \Re v$,

$$
\begin{aligned}
\sigma_{\mathfrak{R}}(g \omega, g v) & =\sup _{r \in I}\left|\frac{g \omega(r)+g v(r)}{2}\right| \\
& =\sup _{r \in I}\left|\int_{0}^{1} \frac{J(r, s, \omega(r))+J(r, s, v(r))}{2} d s\right| \\
& \leq \sup _{r \in I} \int_{0}^{1}\left|\frac{J(r, s, \omega(r))+J(r, s, v(r))}{2}\right| d s \\
& \leq z \sup _{r \in I} \int_{0}^{1}\left|\frac{\omega(r)+v(r)}{2}\right| d s \\
& \leq z \sup _{r \in I}\left(\frac{\omega(r)+v(r)}{2}\right) \int_{0}^{1} d s \\
& \leq z \sigma_{\mathfrak{R}}(\omega, v) .
\end{aligned}
$$

Thus, all the conditions of Theorem 20 are fulfilled. Therefore, the operator $g$ has a unique $\mathrm{fp}$, meaning that the integral equation (58) has a unique solution.

\section{Data Availability}

The data used to support the findings of this study are available from the corresponding author upon request.

\section{Conflicts of Interest}

The authors declare to have no competing interests.

\section{Authors' Contributions}

All authors contributed equally and significantly in writing this article. All authors read and approved the final manuscript.

\section{References}

[1] S. Banach, "Sur les opérations dans les ensembles abstraits et leur application aux équations intégrales," Fundamenta Mathematicae, vol. 3, pp. 133-181, 1922.

[2] S. G. Matthews, "Partial metric topology," Annals of the New York Academy of Sciences, vol. 728, 1 Genseral Topol, pp. 183-197, 1994.

[3] S. Shukla, "Partial b-Metric Spaces and Fixed Point Theorems," Mediterranean Journal of Mathematics, vol. 11, no. 2, pp. 703-711, 2014.
[4] V. Gupta and R. Deep, "Some coupled fixed point theorems in partially ordered S-metric spaces," Miskolc Mathematical Notes, vol. 16, no. 1, pp. 181-194, 2015.

[5] V. Gupta, G. Jungck, and N. Mani, "Some novel fixed point theorems in partially ordered metric spaces," AIMS Mathematics, vol. 5, no. 5, pp. 4444-4452, 2020.

[6] M. Asim, A. Khan, and M. Imdad, "Fixed point results in partial symmetric spaces with an application," Axioms, vol. 8, no. 1, p. 13, 2019.

[7] M. Asim and M. Imdad, "Partial JS-metric spaces and fixed fixed point results," Indian Journal of Mathematics, vol. 61, no. 2, pp. 175-186, 2019.

[8] A. Amini-Harandi, "Metric-like spaces, partial metric spaces and fixed points," Fixed Point Theory and Applications, vol. 2012, no. 1, Article ID 204, 2012.

[9] S. Czerwik, "Contraction mappings in b-metric spaces," Acta Mathematica et Informatica Universitatis Ostraviensis, vol. 1, pp. 5-11, 1993.

[10] A. Branciari, "A fixed point theorem of Banach-Caccioppoli type on a class of generalized metric spaces," Publicationes Mathematiques, vol. 57, pp. 31-37, 2000.

[11] M. Asadi, E. Karapınar, and P. Salimi, "New extension of pmetric spaces with some fixed-point results on $M$-metric spaces," Journal of Inequalities and Applications, vol. 2014, no. 1, Article ID 18, 2014.

[12] N. Mlaiki, A. Zarrad, N. Souayah, A. Mukheimer, and T. Abdeljawed, "Fixed point theorems in $M_{b}$-metric spaces," ournal of Mathematical Analysis and Applications, vol. 7, pp. 1-9, 2016.

[13] M. Asim, I. Uddin, and M. Imdad, "Fixed point results in $M_{v}$ -metric spaces with an application," Journal of Inequalities and Applications, vol. 2019, no. 1, Article ID 280, 2019.

[14] N. Pholasa, S. Antal, I. Uddin, and U. C. Gairola, "Caristi fixed point theorem in $M_{v}$-metric spaces," Dynamic Systems and Applications, vol. 30, no. 3, pp. 439-448, 2021.

[15] N. Y. Özgür, N. Mlaiki, N. Taş, and N. Souayah, "A new generalization of metric spaces: Rectangular $M$-metric spaces," The Mathematical Scientist, vol. 12, no. 3, pp. 223-233, 2018.

[16] P. R. Patle, D. Kumar Patel, H. Aydi, D. Gopal, and N. Mlaiki, "Nadler and Kannan type set valued mappings in $M$-metric spaces and an application," Mathematics, vol. 7, no. 4, p. 373, 2019.

[17] M. E. Gordji, M. Rameani, M. de la Sen, and Y. J. Cho, "On orthogonal sets and Banach fixed point theorem," Fixed Point Theory, vol. 18, no. 2, pp. 569-578, 2017.

[18] H. Baghani and M. Ramezani, "A fixed point theorem for a new class of set-valued mappings in $\mathfrak{R}$-complete (not necessarily complete) metric spaces," Filomat, vol. 31, no. 12, pp. 3875-3884, 2017.

[19] M. E. Gordji and H. Habibi, "Fixed point theory in generalized orthognal metric space," Journal of Linear and Topological Algebra, vol. 6, no. 3, pp. 251-260, 2017.

[20] F. Uddin, K. Javed, H. Aydi, U. Ishtiaq, and M. Arshad, "Control fuzzy metric spaces via orthogonality with an application," Journal of Mathematics, vol. 2021, Article ID 5551833, 12 pages, 2021.

[21] S. Khalehoghli, H. Rahimi, and M. Eshaghi Gordji, "Fixed point theorems in $\mathfrak{N}$-metric spaces with applications," AIMS Mathematics, vol. 5, no. 4, pp. 3125-3137, 2020.

[22] M. U. Ali, Y. Guo, F. Uddin, H. Aydi, K. Javed, and Z. Ma, “On -Partial -Metric Spaces and Related Fixed Point Results with 
Applications," Journal of Function Spaces, vol. 2020, Article ID 6671828, 8 pages, 2020.

[23] K. Javed, F. Uddin, H. Aydi, A. Mukheimer, and M. Arshad, "Ordered-Theoretic Fixed Point Results in Fuzzy b-Metric Spaces with an Application," Journal of Mathematics, vol. 2021, Article ID 6663707, 7 pages, 2021.

[24] A. Alam and M. Imdad, "Relation-theoretic contraction principle," Journal of Fixed Point Theory and Applications, vol. 17, no. 4, pp. 693-702, 2015.

[25] R. D. Maddux, Relation Algebras. Stud. Logic Found. Math., Elsevier B.V, Amsterdam, 2006. 